\section{Duodenal perforation from a pen}

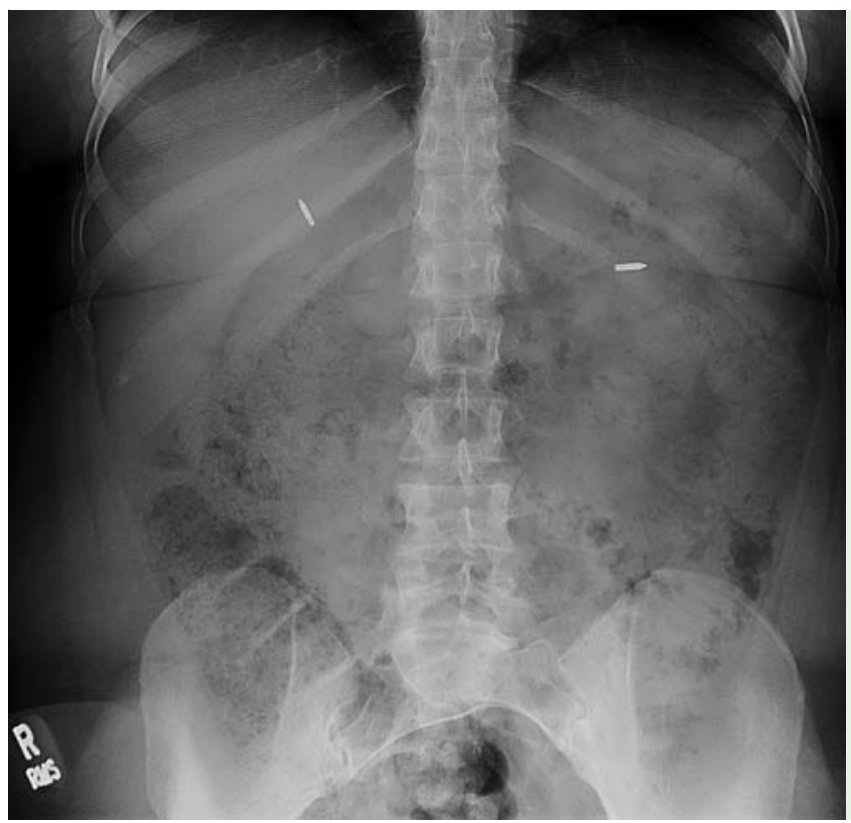

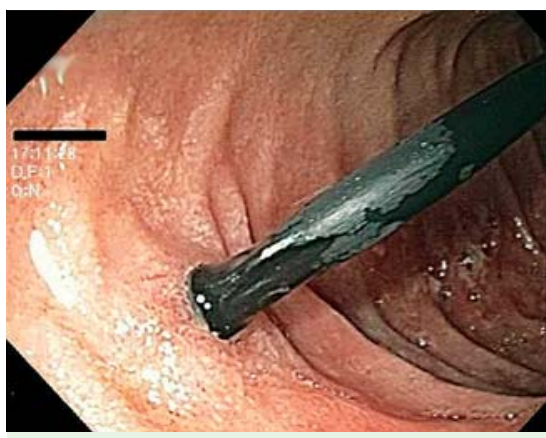

Fig. 2 Endoscopic image showing one of the pen refills perforating through duodenal wall.

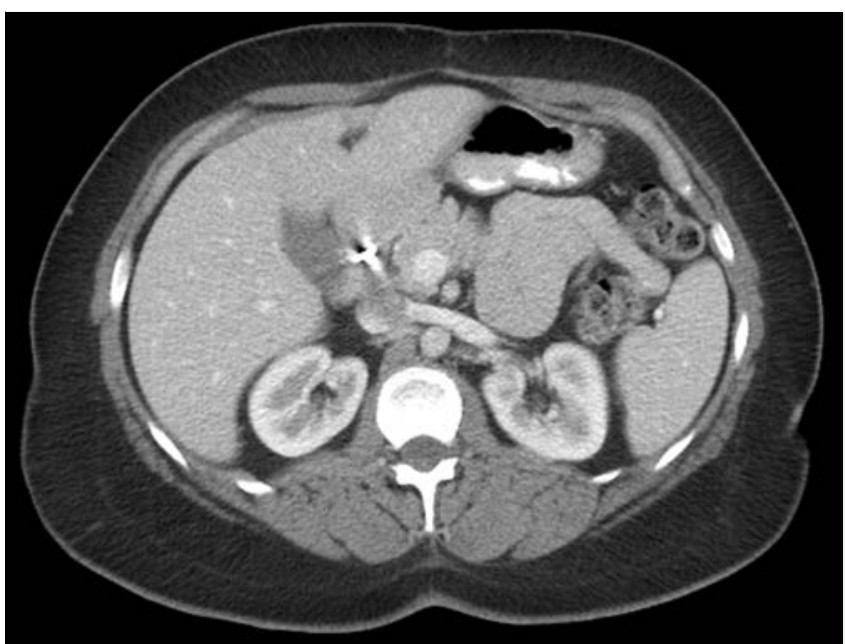

Fig. 1 Abdominal radiograph showing two small, linear, radiopaque foreign bodies resembling the tips of the ingested pen refills.

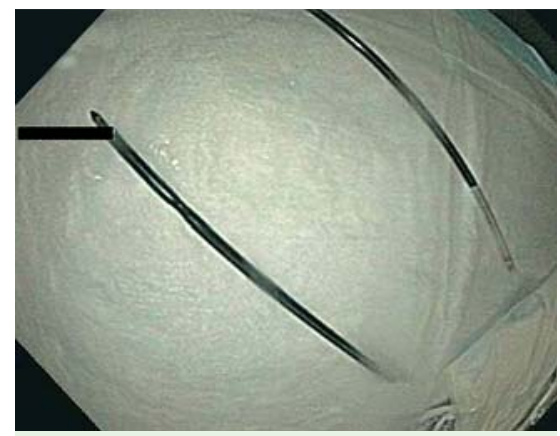

Fig. 3 The two ingested pen refills after their removal.
A 25-year-old woman, who was a longterm resident of a neighboring psychiatric hospital because of schizophrenia, presented to our institution following the ingestion of a foreign body. She had a history of multiple foreign body ingestions and had required 19 esophagogastroduodenoscopies (EGDs) over the previous 18 months. In compliance with the American Society for Gastrointestinal Endoscopy (ASGE) guidelines, not all foreign body ingestions necessitated endoscopic retrieval. Foreign bodies not requiring removal include AA batteries, coins, belt buckles, zippers, and door hinges, among others, while other sharp-pointed objects and those $>6 \mathrm{~cm}$ in length require endoscopic intervention [1].

The patient's current presentation to hospital followed the ingestion of two ballpoint-pen refills 24 hours previously. The positions of the pen refills were confirmed with an abdominal radiograph ( $\bullet$ Fig. 1). Given the length of the foreign bodies, an EGD was arranged. The first object was removed from the stomach with a snare through an overtube. The second object, however, was found to have penetrated deeply through the wall of the duodenal sweep ( Fig.2). Removal of the second pen refill was accomplished by gently pulling it out of the perforated duodenum using a rat-toothed forceps, followed by carefully withdrawing it back into the stomach. A hemostatic clip was successfully placed over the site of the duodenal perforation, and the pen refill was subsequently removed through the overtube ( $\bullet$ Fig. 3).

After the endoscopy, a computed tomography (CT) scan was obtained to check for free air or a fluid collection, neither of which was present ( $\bullet$ Fig.4). The patient was treated with broad-spectrum antibiotics for 1 week, and had no adverse effects.

Endoscopy_UCTN_Code_CCL_1AB_2AF

Competing interests: None

\section{Jana G. Hashash, Lia C. Kaufman, Victor Chedid, Kenneth Fasanella}

Division of Gastroenterology, Hepatology, and Nutrition, Department of Medicine, University of Pittsburgh Medical Center, Pittsburgh, Pennsylvania, USA 


\section{Reference}

1 Ikenberry SO, Jue TL. ASGE Standards of Practice Committee et al. Management of ingested foreign bodies and food impactions. Gastrointest Endosc 2011; 73: 1085-1091

\section{Bibliography}

Dol http://dx.doi.org/

10.1055/s-0034-1391336

Endoscopy 2015; 47: E121-E122

(c) Georg Thieme Verlag KG

Stuttgart · New York

ISSN 0013-726X
Corresponding author

Jana G. Hashash, MD

Division of Gastroenterology, Hepatology, and Nutrition

University of Pittsburgh Medical Center 200 Lothrop Street - PUH, M2, C Wing Pittsburgh

PA 15213

USA

Fax: +1-412-648-9378

alhashashj@upmc.edu 